\title{
Relationship between atrial fibrillatory rate based on analysis of a modified base- apex surface electrocardiogram analysis and the results of transvenous electrical cardioversion in horses with spontaneous atrial fibrillation
}

\author{
Glenn Van Steenkiste, DVM, PhD ${ }^{a, *}$, Jonas Carlson, MD $^{\mathrm{b}}$, \\ Annelies Decloedt, DVM, PhD ${ }^{a}$, Lisse Vera, DVM, PhD ${ }^{a}$, \\ Rikke Buhl, DVM, PhD', Pyotr G. Platonov, $M D, P^{c} D^{d}$, \\ Gunther van Loon, DVM, PhD ${ }^{a}$
}

${ }^{a}$ Equine Cardioteam, Department of Large Animal Internal Medicine, Faculty of Veterinary Medicine, Ghent University, Salisburylaan 133, 9820 Merelbeke, Belgium

${ }^{\mathrm{b}}$ Department of Cardiology, Lund University, 21185 Lund, Sweden

' Department of Veterinary Clinical Sciences, Faculty of Health and Medical Sciences, University of Copenhagen, Højbakkegaard Allé 5, 2630 Taastrup, Denmark

${ }^{\mathrm{d}}$ Arrhythmia Clinic, Skåne University Hospital and Department of Cardiology, Clinical Sciences, Lund University, 21185 Lund, Sweden

Received 31 January 2020; received in revised form 31 December 2020; accepted 5 January 2021

KEYWORDS

Atrial fibrillation; Arrhythmia; Atrial electrophysiology;

Transvenous electrical cardioversion;

\begin{abstract}
Objectives: To compare the atrial fibrillatory rate (AFR) derived from a local right atrial intracardiac recording (RA-FR) and from a single-lead surface electrocardiogram (ECG) during atrial fibrillation (AF) and to evaluate the correlation with transvenous electrical cardioversion (TVEC) threshold (in Joules), number of shocks and cardioversion success rate in horses.

Animals: ECGs and clinical records of horses with AF treated by TVEC. Horses were included if a simultaneous recording of the right atrial intracardiac electrogram and a modified base-apex ECG were available.
\end{abstract}

\footnotetext{
* Corresponding author.

E-mail address: Glenn.VanSteenkiste@ugent.be (G. Van Steenkiste).
} 
Atrial fibrillation cycle length
Materials and methods: Clinical records of horses with AF treated by TVEC were reviewed. Three-minute long episodes of simultaneous electrograms and surface ECG during AF were selected for analysis and compared using Bland-Altman analysis. The mean RA-FR was measured from the deflections on the intracardiac electrogram, while the AFR was extracted from the surface ECG using spatiotemporal QRS and T-wave cancellation.

Results: Seventy-three horses satisfied the inclusion criteria. The mean difference between RA-FR and AFR was -13 fibrillations per minute (fpm), the $95 \%$ limits of agreement were between -66 and $40 \mathrm{fpm}$, and there was a moderate $(\rho=0.65)$ correlation between RA-FR and AFR ( $p<0.001)$. Neither RA-FR nor AFR appeared to influence the TVEC cardioversion threshold or the number of TVEC shocks applied.

Conclusions: The AFR may allow non-invasive long-term monitoring of AF dynamics. Neither RA-FR nor AFR could be used to predict the minimal defibrillation threshold for TVEC.

(C) 2021 Elsevier B.V. All rights reserved.

\section{Introduction}

Atrial fibrillation $(\mathrm{AF})$ is the most common pathological arrhythmia in horses with an estimated prevalence of $2.5 \%$ [1]. Medical therapy using quinidine sulphate, as well as transvenous electrical cardioversion (TVEC) are well established treatments $[2,3]$. The recurrence rate of AF after successful treatment is between 15 and $39 \%$ at 1 year after conversion $[4,5]$. The atrial effective refractory period is an important variable related to the degree of atrial electrical remodelling [6,7]. This variable has been studied in horses using programmed electrical stimulation, but the technique can only be applied during sinus rhythm [8]. During $\mathrm{AF}$, the atrial fibrillation cycle length (AFCL) can be used instead as it is directly related to the atrial effective refractory period. In people with AF, the AFCL has been used to investigate the effects of antiarrhythmic drugs on AF [9-11] and has been identified as an independent factor for prediction of $\mathrm{AF}$ treatment outcome and AF recurrence rate [10]. In horses, no independent correlation was found between the AFCL and AF recurrence, but correlations were found between the $A F C L$ and $A F$ duration
Abbreviations
AF atrial fibrillation
AFCL atrial fibrillation cycle length
AFR atrial fibrillatory rate
ECG electrocardiogram
RA-FR atrial fibrillatory rate derived from a local right atrial intracardiac recording
TVEC transvenous electrical cardioversion

and also between the shortest $5 \% \mathrm{AFCL}$ to left atrial size ratio and AF recurrence [7]. Because horses with a longer AF duration are less likely to convert using a medical treatment [12], the AFCL could be a useful indicator to recommend TVEC. In experimentally pacing-induced $\mathrm{AF}$, only horses with an atrial fibrillatory rate (AFR) less than 350 fibrillations per minute (fpm) showed spontaneous cardioversion to SR [13] but no similar data are published from horses with naturally occurring paroxysmal AF.

The AFCL is typically measured invasively from bipolar intra-atrial electrogram recordings as the time between two successive atrial depolarisations. Tissue Doppler has been validated as a noninvasive way of determining the AFCL in horses and humans, but this requires specialized equipment, an experienced operator and cannot be used for long-term continuous monitoring [14,15]. Another non-invasive option is deriving the AFR from the surface electrocardiogram (ECG), a technique that has been validated against the intracardiac AFCL recordings in humans [16]. Although the technique could not be used to estimate the exact local AFCL within the atrium, it was a good representation of the dynamics of the AFCL. Measuring the AFR from the surface ECG would allow long-term monitoring, for example, to investigate the effects of drugs on AF in horses. Recently, the effect of antiarrhythmic drugs on the surface ECG-derived AFR was studied in horses $[13,17]$. However, so far the technique has not been compared and validated with the intracardiac AFCL in horses.

The first goal of this study was to compare the non-invasively determined AFR from the surface ECG with the AFCL derived from a right atrial electrogram in horses with naturally occurring $\mathrm{AF}$ using the described technique. The second aim 


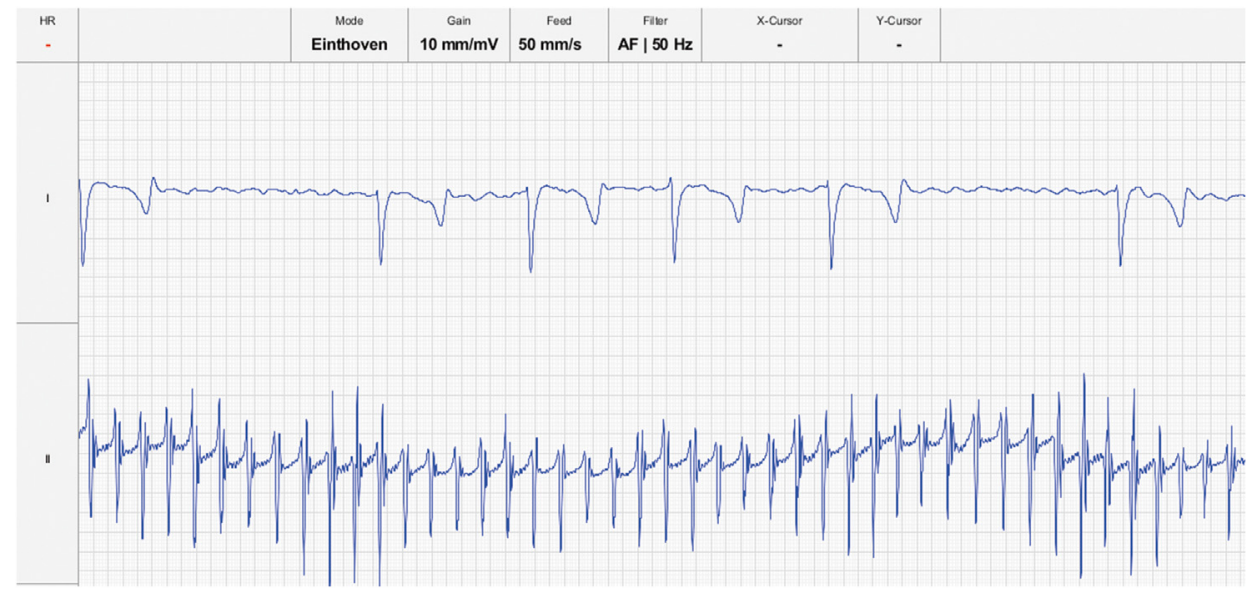

Fig. 1 Example of an intra-atrial electrogram (lower trace) from the right atrium recorded simultaneously with a modified base-apex surface electrocardiogram (upper trace). The mean intra-atrial fibrillation rate is calculated from the time interval between 2 successive atrial depolarisations.

was to evaluate if the TVEC energy threshold, number of shocks needed for conversion, and successful conversion were related to the AFR.

\section{Animals, materials and methods}

\section{Study population}

Records of horses referred to the Department of Large Animal Internal Medicine, Ghent University, for TVEC of AF since 2013 were reviewed to identify horses in which at least three minutes of good quality, simultaneous recording of the intra-atrial electrogram and surface ECG under sedation, before the TVEC procedure, were available. The following characteristics from the TVEC procedure were collected: restoration of sinus rhythm (yes, no), energy delivery at cardioversion (Joules) and number of shocks needed for cardioversion. The AF duration was not available in the current data set. The TVEC procedure was performed as previously described [3].

\section{Intracardiac electrogram recordings}

The TVEC procedure started with insertion of catheters in the standing, sedated (detomidine $7.5 \mu \mathrm{g} / \mathrm{kg}$ IV, Domidine ${ }^{\circledR}{ }^{\mathrm{e}}$ ) horse. As part of our standard procedure, a temporary pacing catheter, connected to an adapted Televet 100 device $^{f}$, was first inserted in the right atrium and positioned at the intervenous tubercle. Subsequently, two TVEC catheters were inserted and positioned in left pulmonary artery and right ventricle under ultrasound guidance. During TVEC catheter positioning the

\footnotetext{
e Eurovet Animal Health B.V., Bladel, The Netherlands.

${ }^{f}$ Engel Engineering Services GmbH, Heusen-stamm, Germany.
}

Televet 100 device recorded the right atrial electrogram together with a modified base-apex ECG as shown in Fig. 1. From the intracardiac recording, the AFCL was determined using a custom-made script in Matlab R2018b ${ }^{\mathrm{g}}$. The script divided the intracardiac electrogram into separate one-minute recordings and applied a peak detection algorithm that identified all positive deflections within each electrogram. All electrogram traces were visually checked by the operator (GVS) for correct peak detection. In case the quality was insufficient, the one-minute intra-atrial trace and corresponding surface ECG trace were rejected and not included in the study and another one-minute good quality trace was selected from the same horse. The AFCL, defined as the time interval between two consecutive intraatrial depolarisations, was averaged over each one minute of recording to calculate a global average over the three minutes.

\section{Electrocardiogram recordings and signal processing}

The modified base-apex ECG (negative electrode at right withers, positive electrode left thorax caudal to the olecranon) was recorded simultaneously with the intra-atrial electrogram. Only the data of the surface ECG lead were exported to a comma separated values file for processing using the AFR Tracker software ${ }^{\text {h }}$ to obtain the AFR in fpm averaged over each one-minute segment, to calculate a global average over the three minutes. This software uses spatiotemporal QRS and T-wave cancellation and has been applied to equine ECGs

\footnotetext{
${ }^{g}$ Mathworks, Natick, United States.

${ }^{\text {h }}$ CardioLund Research AB, Lund, Sweden.
} 
Table 1 Descriptive statistics (of mean, median, standard deviation, and range) for atrial fibrillatory rate, total cardioversion energy, and number of shocks in 73 horses with atrial fibrillation.

\begin{tabular}{lcccc}
\hline Measurement & Mean & Median & s.d. & Range \\
\hline RA-FR (fpm) & 356 & & 23 & $306-410$ \\
AFR (fpm) & 369 & & 38 & $303-527$ \\
Energy (Joules) & & 200 & & $150-360$ \\
Number of shocks & & 1.5 & & $1-15$ \\
\hline
\end{tabular}

s.d., standard deviation; RA-FR, intracardiac atrial fibrillation rate; AFR, surface ECG atrial fibrillatory rate; fpm, fibrillations per minute.

previously [13,16-18]. After detection of the QRS complexes and T waves, the software calculates a medium QRS-T complex from each one-minute segment and subtracts this from the segment leaving a residual ECG. From this residual ECG, the software determines the average dominant frequency over time as the AFR.

\section{Statistics}

All statistics were performed using R v3.4.4 [19]. The level of significance was $p=0.05$. All data were tested for normality by visual control of the frequency histogram and Q-Q plot. Data are reported as means (SD) or medians (range) appropriate for the distribution of the data. For statistical comparison between the intra-atrial AFCL and the AFR from the surface ECG, the intra-atrial $A F C L$ was converted to $\mathrm{fpm}$ instead of milliseconds. The Bland-Altman mean bias, $95 \%$ limits of agreement and Pearson correlation were calculated. A paired ttest was used to test the significance of the mean difference between both methods. The Spearman correlation was calculated between the AFR and energy (Joules) needed for conversion. A zerotruncated negative binomial regression was used to assess if there was significant effect of the AFR on the number of shocks needed for cardioversion.

\section{Results}

Seventy-three horses met the inclusion criteria: 60 warmbloods, 9 trotters and 4 thoroughbreds; 11 stallions, 43 geldings and 19 mares; mean body weight $581 \pm 79 \mathrm{~kg}$ and mean age $9.5 \pm 4$ years. Intracardiac ECG recordings from 19 of these horses have also been reported in another study [7]. Both the fibrillation rate derived from a local right atrial intracardiac recording (RA-FR) and AFR were normally distributed while the number of shocks and energy needed for conversion were not normally distributed. Median [range] for non-normal distributed and mean with standard deviation for normal distributed values are shown in Table 1. A scatter plot of the RA-AFR versus AFR is shown in Fig. 2A. The RA-FR was significantly lower compared to the AFR $(p<0.001$, power $=0.98)$ and both were moderately correlated $(\rho=0.65, p<0.001)$. Bland-Altman mean difference was $-13 \mathrm{fpm}$, the $95 \%$ limits of agreement were between -66 and $40 \mathrm{fpm}$, and the BlandAltman plot is shown in Fig. 2B. Correlations, 95\% confidence intervals and $\mathrm{p}$-values are shown in Table 2. In total $71(97 \%)$ out of 73 horses did convert to sinus rhythm following TVEC. Because only 2 horses did not convert to SR, data were not further analysed statistically. No significant correlation could be found between the RA-FR or AFR and the amount of energy needed for cardioversion to SR. No significant effect of the RA-FR or AFR could be found on the number of shocks needed for cardioversion.
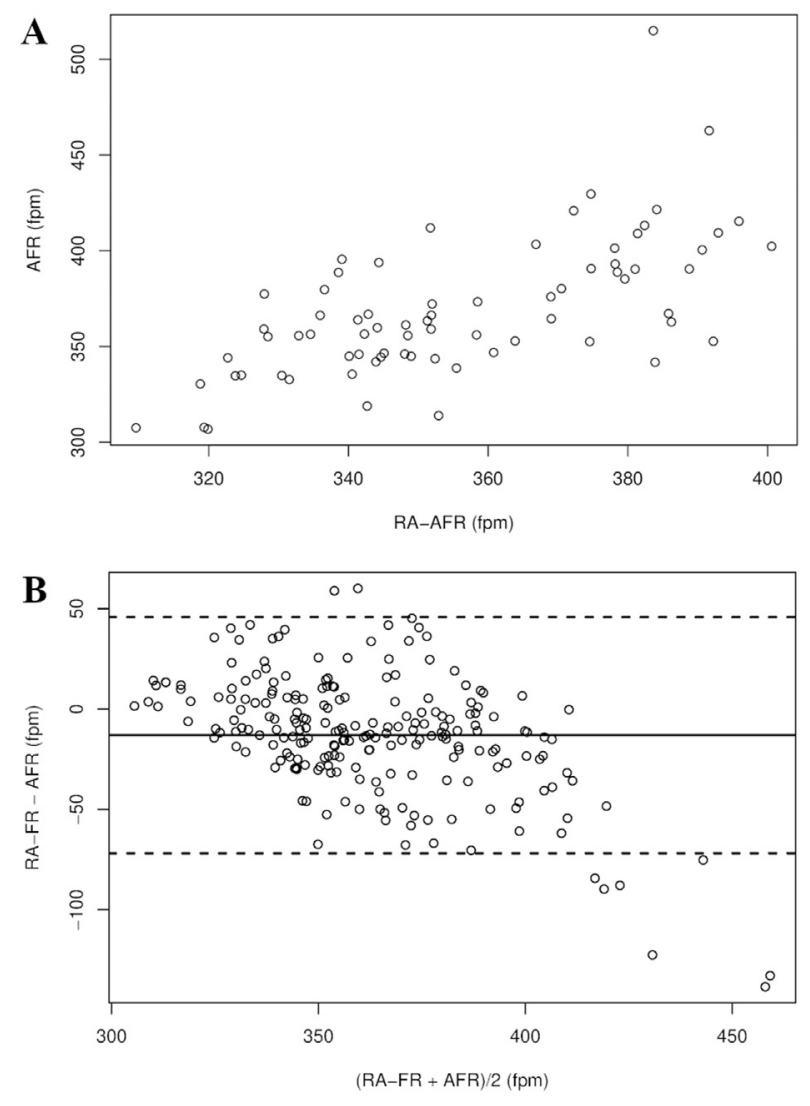

Fig. 2 Panel A: scatter plot comparing the intra-atrial right atrial fibrillatory rate (RA-FR) and the surface electrocardiogram atrial fibrillatory rate (AFR) in fibrillations per minute (fpm). Each point represents the average atrial fibrillation rate over $3 \mathrm{~min}$ in a single horse. Panel $B$ is a Bland-Altman plot of agreement between the intraatrial right atrial fibrillatory rate (RA-FR) and the surface electrocardiogram atrial fibrillatory rate (AFR). The solid line represents the bias, the upper and lower lines represent the upper and lower 95\% limits of agreement. 
Table 2 Correlation and regression coefficients, 95\% confidence intervals and p-values of the statistical analysis of the atrial fibrillatory rate, total cardioversion energy and number of shocks in 73 horses who were treated for atrial fibrillation.

\begin{tabular}{|c|c|c|c|c|}
\hline Measurement & Correlation & Regression coefficient & $95 \%$ Confidence interval & $p$-value \\
\hline \multicolumn{5}{|l|}{ RA-FR } \\
\hline AFR & $0.65^{\mathrm{a}}$ & & -6640 & $<0.001$ \\
\hline $\begin{array}{l}\text { Number } \\
\text { of shocks }\end{array}$ & & $0.014^{\mathrm{b}}$ & & 0.262 \\
\hline Energy & $0.12^{c}$ & & & 0.325 \\
\hline \multicolumn{5}{|l|}{ AFR } \\
\hline $\begin{array}{l}\text { Number } \\
\text { of shocks }\end{array}$ & & $0.003^{2}$ & & 0.692 \\
\hline Energy & $0.13^{c}$ & & & 0.288 \\
\hline
\end{tabular}

\section{Discussion}

The present study shows a significant correlation between the AFR measured from a surface ECG and the RA-FR measured from a right atrial recording, but also a significant difference and wide limits of agreement between both methods for measuring the AFR.

Recent studies in horses with experimentally induced AF have shown that the AFR dynamics are similar in horses compared to humans with paroxysmal AF and that AFR could be used to study the effect of antiarrhythmic drugs during AF. However, the AFR was not compared to the RA-FR in these studies $[13,17]$. A comparison between manual calculation of the $f-f$ interval from the surface ECG and intracardiac RA AFCL found a moderate correlation between both methods'. However, these results were possibly biased towards longer $f-f$ intervals, and thus segments with course $\mathrm{AF}$ on the ECG, because otherwise $f$ waves were not clearly identifiable.

The absolute mean difference between RA-FR and AFR in our study (13 fpm) is similar compared to a study in human medicine (11 fpm), but with larger limits of agreement [16]. Similar as in human medicine, the AFR is not an exact measurement of the local AFCL within the atrium, but it is rather a representation of global AF dynamics. The correlation between RA-FR and AFR supports the potential to use AFR to monitor long-term dynamics of $\mathrm{AF}$ [13]. In addition to tracking the

\footnotetext{
I De Clercq D, van Loon G, Deprez P. Determination and correlation of $f-f$ interval and right atrial fibrillation cycle length in 30 horses with atrial fibrillation. J. Vet. Intern. Med., Barcelona, Spain: 2009, p. 10-1.
}

effect of antiarrhythmic compounds on $A F$, the technique could also be used to assess the effect of circadian rhythm or autonomic tone on AF dynamics. Because the AFR does not require long RR intervals to be measured, in contrast to tissue Doppler, the AFR could also be used at higher heart rates, such as during exercise [15]. Only global AF dynamics can be estimated with the AFR since the technique currently does not allow for local measurements, although these are possible with tissue Doppler imaging and intracardiac electrogram measurements.

Atrial fibrillation cycle length differences between intra-atrial electrogram recording and tissue Doppler image analyses have been documented in a previous study in horses, but these were attributed to spatiotemporal AFCL variation because both measurements were not performed simultaneously and not at the same intra-atrial location. In our study, recordings of both surface ECG and intracardiac electrograms were carried out simultaneously, avoiding the temporal variable. This leaves the spatial AFCL variation, which could not be avoided due to the electrode configuration of a modified base-apex ECG. In horses with naturally occurring persistent $A F$, a significantly faster AFR in the left atrium compared to the right atrium has been demonstrated [15]. The AFR in the present study was significantly faster compared to the RA-FR, which might suggest that the modified base-apex lead is more influenced by left compared to right atrial electrical activity.

In our study the modified base-apex lead (bipolar) was used for recording the surface ECG. For this electrode configuration, the electrodes are positioned at a large distance from the atria and encompass a much larger portion of the atria 
compared to the unipolar $\mathrm{V}_{1}$ or oesophageal lead used in human trials $[16,20]$. Because of the long distance between recording electrodes and atria, this lead probably results in a more global AFR measurement compared to the more localized AFR from $V_{1}$ in human medicine. The latter has a better relationship with the fibrillatory rate of the coronary sinus and left pulmonary veins in comparison with right atrial fibrillatory rate $[16,21]$. The larger the anatomical distance between the intracardiac and surface ECG recording location, the bigger the difference between the intracardiac $\mathrm{AF}$ rate and AFR. This is also represented by the wide limits of agreement between both methods and the relatively low shared variability of $42 \%$. These theories should be further investigated by applying different electrode configurations, using unipolar electrodes close to the right atrial free wall, or using intraatrial recordings from the left atrium which could be compared to both the global AFR and a unipolar AFR. A transseptal atrial puncture is used to record left atrial electrograms in people, but this technique has not yet been described in horses. Another option would be to record left atrial AFCL from the coronary sinus since this approach does not require access to the left atrium. Despite the relatively weak correlation between both methods in the present study, the AFR derived from the surface ECG can still be useful for long-term monitoring of the AFR as was demonstrated by a previous study in horses [13, 17].

As only 2 out of 73 horses did not convert to sinus rhythm, some conclusions could not be drawn from this data set. No significant effect was found of the RA-FR or AFR on the number of shocks or the energy requirements needed for cardioversion were identified. This is in contradiction to a human studies in which a moderate to strong positive correlation was found between the minimal threshold for external cardioversion and AFR $[22,23]$. This may indicate that other mechanisms play a role in the minimal cardioversion threshold such as atrial structural defects or atrial size. Although pacing induced AF in horses yielded similar results as in humans, it remains unclear if pacing induced $A F$ reflects naturally occurring $A F$ in horses [8].

\section{Limitations}

We only measured electrograms at one right atrial location, thus spatial intracardiac fibrillatory rate variability and correlation with the AFR could not be assessed. In addition, it is unclear if recording in the right atrium is a good surrogate for overall atrial activation due to potential interatrial differences in fibrillatory rate. Because only one surface ECG lead was recorded, no information is available about the use of different lead configurations on the AFR as this might have improved the correlation between both methods. The current measurements were done with sedation. Detomidine has been reported to significantly decrease AFR, but the decrease was very small and the measurement can therefore be considered a reliable indicator for atrial electrical remodelling [24].

\section{Conclusion}

The surface ECG-derived AFR had a shared variability of $42 \%$ with the intracardiac RA-FR. The non-invasive approach may allow long-term monitoring of the AFR in order to investigate AF pathophysiology in horses and the effect of antiarrhythmic drugs. However, results show a significantly faster AFR compared to the RA-FR and wide limits of agreement. Neither RAFR nor AFR could be used to predict the minimal defibrillation threshold for TVEC.

\section{Conflicts of interest}

None of the authors have any conflicts of interest or financial ties to disclose.

\section{Acknowledgements}

G.V.S. and L.V. are PhD fellows of the Research Foundation Flanders, grant $1 \mathrm{~S} 56217 \mathrm{~N}$ and $1134917 \mathrm{~N}$, respectively. This research was supported by the Special Research Fund Ghent University, grant BOF.BAS.2018.0022.01. The authors would like to acknowledge Inge Van Damme for her statistical assistance on this study.

\section{References}

[1] Else RW, Holmes JR. Pathological changes in atrial fibrillation in the horse. Equine Vet J 1971;3:56-64.

[2] Reef VB, Reirner JM. Spencer $P$ a. Treatment of atrial fibrillation in Horses: new perspectives. J Vet Intern Med 1995;9:57-67.

[3] De Clercq D, van Loon G, Schauvliege S, Tavernier R, Baert K, Croubels S, De Backer P, Deprez P. Transvenous electrical cardioversion of atrial fibrillation in six horses using custom made cardioversion catheters. Vet J 2008; 177:198-204.

[4] McGurrin MKJ, Physick-Sheard PW, Kenney DG. Transvenous electrical cardioversion of equine atrial fibrillation: patient factors and clinical results in 72 treatment episodes. J Vet Intern Med 2008;22:609-15. 
[5] Decloedt A, Schwarzwald CC, De Clercq D, Van Der Vekens N, Pardon B, Reef VB, van Loon G. Risk factors for recurrence of atrial fibrillation in horses after cardioversion to sinus rhythm. J Vet Intern Med 2015;29: 946-53.

[6] Wijffels MCEF, Kirchhof CJHJ, Dorland R, Allessie MA. Atrial fibrillation begets atrial fibrillation. Circulation 1995;92:1954-68.

[7] De Clercq D, Decloedt A, Sys SU, Verheyen T, Van Der Vekens N, van Loon G. Atrial fibrillation cycle length and atrial size in horses with and without recurrence of atrial fibrillation after electrical cardioversion. J Vet Intern Med 2014;28:624-9.

[8] van Loon G, Duytschaever M, Tavernier R, Fonteyne W, Jordaens L, Deprez P. An equine model of chronic atrial fibrillation: methodology. Vet J 2002;164:142-50.

[9] Drewitz I, Willems S, Salukhe TV, Steven D, Hoffmann BA, Servatius H, Bock K, Aydin MA, Wegscheider K, Meinertz T, Rostock T. Atrial fibrillation cycle length is a sole independent predictor of a substrate for consecutive arrhythmias in patients with persistent atrial fibrillation. Circ Arrhythm Electrophysiol 2010;3:351-60.

[10] Meurling CJ, Roijer A, Waktare JEP, Holmqvist F, Lindholm CJ, Ingemansson MP, Carlson J, Stridh M, Sörnmo L, Olsson SB. Prediction of sinus rhythm maintenance following $\mathrm{DC}$-cardioversion of persistent atrial fibrillation - the role of atrial cycle length. BMC Cardiovasc Disord 2006;6:11.

[11] Haïssaguerre M, Sanders P, Hocini M, Hsu L-F, Shah DC, Scavée C, Takahashi Y, Rotter M, Pasquié J-L, Garrigue S, Clémenty J, Jaïs P. Changes in atrial fibrillation cycle length and inducibility during catheter ablation and their relation to outcome. Circulation 2004;109: 3007-13.

[12] Reef VB, Levitan CW, Spencer PA. Factors affecting prognosis and conversion in equine atrial fibrillation. $\mathrm{J}$ Vet Intern Med 1988;2:1-6.

[13] Hesselkilde EZ, Carstensen H, Haugaard MM, Carlson J, Pehrson S, Jespersen T, Buhl R, Platonov PG. Effect of flecainide on atrial fibrillatory rate in a large animal model with induced atrial fibrillation. BMC Cardiovasc Disord 2017; 17:289.

[14] Duytschaever M, Heyse A, De Sutter J, Crijns H, Gillebert T, Tavernier R, Tieleman R. Transthoracic tissue Doppler imaging of the atria: a novel method to determine the atrial fibrillation cycle length. J Cardiovasc Electrophysiol 2006;17:1202-9.

[15] Decloedt A, De Clercq D, van der Vekens N, Verheyen T, van Loon G. Noninvasive determination of atrial fibrillation cycle length by atrial colour tissue Doppler imaging in horses. Equine Vet J 2014;46:174-9.

[16] Holm M, Pehrson S, Ingemansson $M$, Sörnmo $L$, Johansson R, Sandhall L, Sunemark M, Smideberg B, Olsson C, Olsson SB. Non-invasive assessment of the atrial cycle length during atrial fibrillation in man: introducing, validating and illustrating a new ECG method. Cardiovasc Res 1998;38:69-81.

[17] Carstensen H, Kjœr L, Haugaard MM, Flethøj M, Hesselkilde EZ, Kanters JK, Pehrson S, Buhl R, Jespersen T. Antiarrhythmic effects of combining dofetilide and ranolazine in a model of acutely induced atrial fibrillation in horses. J Cardiovasc Pharmacol 2018;71:26-35.

[18] Stridh M, Sommo L. Spatiotemporal QRST cancellation techniques for analysis of atrial fibrillation. IEEE Trans Biomed Eng 2001;48:105-11.

[19] Team RC. R: a language and environment for statistical computing. 2019.

[20] Bollmann A, Kanuru N, McTeague K, Walter P, DeLurgio D, Langberg J. Frequency analysis of human atrial fibrillation using the surface electrocardiogram and its response to ibutilide. Am J Cardiol 1998;81:1439-45.

[21] Husser D, Stridh M, Sornmo L, Olsson SB, Bollmann A. Frequency analysis of atrial fibrillation from the surface electrocardiogram. Indian Pacing Electrophysiol J 2004;4: 122-36.

[22] Tai CT, Chen SA, Liu AS, Yu WC, Ding YA, Chang MS, Kao T. Spectral analysis of chronic atrial fibrillation and its relation to minimal defibrillation energy. PACE - Pacing Clin Electrophysiol 2002;25:1747-51.

[23] Bollmann A, Mende M, Neugebauer A, Pfeiffer D. Atrial fibrillatory frequency predicts atrial defibrillation threshold and early arrhythmia recurrence in patients undergoing internal cardioversion of persistent atrial fibrillation. PACE - Pacing Clin Electrophysiol 2002;25:1179-84.

[24] Decloedt A, De Clercq D, van der Vekens N, Verheyen T, Ven S, van Loon G. Influence of detomidine on atrial fibrillation cycle length measured by intracardiac electrogram recording and by colour tissue Doppler imaging in horses. Equine Vet J 2016;48:21-6. 